\title{
Language-Related Field Potentials in the Anterior-Medial Temporal Lobe: I. Intracranial Distribution and Neural Generators
}

\author{
Gregory McCarthy, ${ }^{1,2}$ Anna C. Nobre, ${ }^{1,2, a}$ Shlomo Bentin, ${ }^{3}$ and Dennis D. Spencer ${ }^{2}$ \\ 'Neuropsychology Laboratory, VA Medical Center, West Haven, Connecticut 06516, ${ }^{2}$ Section of Neurosurgery, Yale \\ University School of Medicine, New Haven, Connecticut 06510, and ${ }^{3}$ Department of Psychology, Hebrew University, \\ Jerusalem, Israel
}

\begin{abstract}
Field potentials were recorded from intracranial electrodes in humans to study language-related processing. Subjects viewed sentences in which each word was presented successively in the center of a video monitor. Half of the sentences ended normally, while the other half ended with a semantically anomalous word. The anomalous sentenceending words elicited a large negative field potential with a peak latency near 400 msec, which was focally distributed bilaterally in the anterior medial temporal lobe (AMTL), anterior to the hippocampus and near the amygdala. Subdural electrodes positioned near the collateral sulcus just inferior and lateral to the amygdala recorded a positive field potential at the same latency. This spatial distribution of voltage suggested that this language-sensitive field potential was generated in the neocortex near the collateral sulcus and anterior fusiform gyrus. Additional task-related field potentials were recorded in the hippocampus. The AMTL field potential at $\mathbf{4 0 0}$ msec shares characteristics with the $\mathbf{N 4 0 0}$ potential recorded from scalp electrodes that has been associated with semantic processing.
\end{abstract}

The investigation of the neural organization and temporal sequence of language processing in the brain has been aided by noninvasive neurophysiological studies in which field potentials have been recorded from arrays of scalp electrodes (Kutas and Hillyard, 1980, 1989; Bentin et al., 1985; Neville et al., 1986). These studies have revealed a sequence of event-related potentials (ERPs) elicited by words with distinct spatial distributions reflective of their underlying neural substrate (Nobre and McCarthy, 1994). One such ERP, termed N400 for its negative polarity and approximate peak latency of $400 \mathrm{msec}$, can be elicited readily by words when presented without appropriate semantic context in sentence and word processing tasks (Kutas and Hillyard, 1980). N400 has attracted considerable attention

\footnotetext{
Received Mar. 22, 1994; revised June 23, 1994; accepted July 6, 1994.

We thank Dr. Truett Allison, Dr. Marsel Mesulam, and two anonymous reviewers for constructive criticism of the manuscript, and Kevin McCarthy, Elizabeth Roessler, and Marie Luby for technical assistance. Dr. Marta Kutas provided some of the experimental stimuli. Support for this research was provided by the Department of Veteran's Affairs, NIH Grant MH05286, and the US-Israel Binational Science Foundation.

Correspondence should be addressed to Gregory McCarthy, Ph.D., Neuropsychology Laboratory, 116 Bl, VA Medical Center, West Haven, CT 06516.

a Present address: Department of Experimental Psychology, Oxford University, South Parks Road, Oxford, OX1 3UD, UK

Copyright (c) 1995 Society for Neuroscience $0270-6474 / 95 / 151080-10 \$ 05.00 / 0$
}

because subsequent experiments have linked its appearance to the access of the lexical or semantic representation of a word (Kutas and Van Petten, 1988; Fischler and Raney, 1989) or, alternatively, to associative processes that integrate word representations with current context (Rugg and Doyle, 1992; Holcomb, 1993). More recent experiments have demonstrated that meaningful nonverbal stimuli (such as objects and familiar faces) elicit ERPs with similar latency and waveform (Barrett and Rugg, 1989, 1990; Holcomb and McPherson, 1994), raising the possibility that N400 may represent neural activity associated with a general process of activating and/or modifying semantic representations.

While noninvasive scalp recordings have contributed greatly to an explication of the psychological processes represented by $\mathrm{N} 400$, they have not been equally successful in identifying its neural substrate. Electrical field potentials recorded from intracranial electrodes have the spatial resolution required to make inferences about the neuronal ensembles and anatomical structures involved in perceptual and cognitive processing (e.g., Halgren et al., 1980; Allison et al., 1994). Previous studies have shown that field potentials elicited during language tasks can be recorded from medial temporal lobe in patients with intracranial depth electrodes (McCarthy and Wood, 1984; McCarthy et al., 1985; Smith et al., 1986). Smith et al. (1986) presented recordings from 10 patients performing language tasks, including a recognition memory task using visually presented words. A field potential with a $460 \mathrm{msec}$ mean latency was elicited by words, and was attenuated when the words were repeated. This potential was negative in the region of the amygdala, similar to the ERP rcported in preliminary studies by McCarthy and colleagues (McCarthy and Wood, 1984; McCarthy et al., 1985). Smith et al. also reported that this ERP inverted in polarity in the region of the anterior hippocampus, implicating the hippocampus as its neural generator. Smith et al. further showed that the amplitude of this potential was strongly modulated by prior experience with the eliciting word, and suggested that the neural activity was associated with recent event memory formation.

In this study, we sought to identify the brain region or regions whose neurons generate the scalp-recorded N400 using the benchmark anomalous sentence task in which N400 was first described (Kutas and Hillyard, 1980). We demonstrate that a negative field potential in the anterior medial temporal region (AMTL N400) can be reliably elicited by semantically anomalous sentence-ending words. In the following article (Nobre and McCarthy, 1995), we examinc the role of word meaning and semantic context in the appearance of this AMTL N400. 


\section{Materials and Methods}

Subjects. Sixty-four patients ( 32 females) with complex partial epilepsy participated. These patients were being evaluated for resective surgery to relieve medically intractable seizures at the Yale-West Haven VAMC epilepsy surgery program. As part of this evaluation, multicontact depth probes and subdural electrode strips were implanted to localize their seizure focus or foci. Experimental recordings were obtained concurrently with continuous computerized EEG monitoring 3-10 d following implant surgery. The experimental nature of the task was explained to each patient, who indicated voluntary participation by signing an informed consent document. The protocols used in these experiments were approved by the Yale and VA Human Investigations Committees. The patients ranged in age from 16 to 46 years (mean age was 29.8 years). The mean verbal IQ was 93.4 and ranged from 70 to 130 . Handedness information was available for 53 patients, of whom 46 were right handed.

Stimuli. The stimuli were 160-240 sentences, each composed of 615 words. The words varied between 1 and 12 characters in length. Each sentence was constructed so that there was a single best completion for its terminal word. Half of the sentences (semantically NORMAL) terminated with this best choice (e.g., when you go to bed turn off the living room lights.). The remaining half (semantically aNOMALOUs) terminated with a syntactically correct, but semantically inappropriate word (e.g., I ordered a ham and cheese scissors.). NORMAL and ANOMALOUs sentences were intermixed randomly in the experimental list.

Procedure. The study was conducted in the paticnt's hospital room which was dimly lighted during the experiment. The patient reclined upright in an adjustable bed and faced a computer monitor approximately $70 \mathrm{~cm}$ distant. The words in each sentence were displayed individually and in rapid succession as red characters upon a white rectangular background in the center of a dark screen. Each character subtended approximately $0.42^{\circ}$ of visual angle, and the average word subtended $2.4^{\circ}$. The duration of each word was set to $200 \mathrm{msec}$ and the interval between word onsets was set to $500 \mathrm{msec}$. If a patient had difficulty reading at that rate, the interval between words was increased up to $800 \mathrm{msec}$. The last word of the sentence was indicated by a period. After an interval of $3-4 \mathrm{sec}$, the character string "XXXXX" appeared, which marked the onset of the next sentence. The patients were instructed to read each sentence silently for comprehension and were told that some sentences might appear odd. No behavioral mcasures were obtained, but informal debriefing ensured that patients had read the sentences.

Electrode placement and localization. Recordings were made from a total of 3676 electrodes. The typical recording array included 32-64 electrodes chosen from bilaterally placed posterior-temporal (PT) depth probes and 2-14 subdural electrode strips in each patient. The PT probes were inserted approximately $25 \mathrm{~mm}$ from the midline and superior to the calcarine sulcus and were targeted to pass through the hippocampus along its longitudinal axis and to terminate in the anterior temporal lobe within the amygdala. Additional probes were placed in some patients, including a midtemporal (MT) probe that entered anterior to premotor cortex and passed into the anterior medial temporal lobe near the amygdala. Subdural electrode strips were inserted through burr holes in the skull and aimed at specific neocortical regions. Subdural strips consisted of 4-16 stainless steel electrode contacts, each $2.2 \mathrm{~mm}$ in diameter and embedded in silastic. The interelectrode spacing was 1 $\mathrm{cm}$. In all figures, the electrode contact most distal to the insertion point is numbered 1 .

Electrode materials, stereotaxic procedures, and methods for postoperatively localizing electrodes evolved over the duration of this study. Details concerning these procedures can be found in McCarthy et al. $(1989,1991)$. For the first 39 subjects (19 females), depth probes were constructed of 18 laminated platinum-iridium wires epoxied to needle stock. Each wire had an uninsulated $1 \mathrm{~mm}$ platinized section that served as the electrode contact. The electrode contacts were equidistantly spaced along the probes, which were stereotaxically inserted through steel guide pins. The probe lengths and, consequently, interelectrode distances, varied across patients from 3.3 to $6.1 \mathrm{~mm}$. The probe trajectorics and electrode positions were determined from skull films obtained with the individual in the stereotaxic frame (Darcey and Williamson, 1985). Bony landmarks were used to project the electrode contacts into the proportional system of Talairach and Szikla (1967).

For the remaining 25 subjects ( 13 females), the probes were inserted using a MRI-guided stereotaxic system that allowed previewing each probe's trajectory in three dimensions. The probes were composed of a $1 \mathrm{~mm}$ diameter soft, hollow silastic core with 12 cylindrical nichrome electrodes spaced equally from the distal tip. A flexible stylet was placed in the hollow core for insertion of the probe, and was subsequently removed. The locations of the probe and subdural strip electrodes were determined from their susceptibility artifacts in postoperative MRIs. The localization of electrodes was facilitated by software that displayed each electrode in axial, coronal, sagittal, and reformatted oblique views. A total of 1376 electrode locations were determined in this manner. For each of these electrodes, Talairach coordinates were also computed (Talairach and Tournoux, 1988).

Field potential recordings. EEG was recorded from 32-64 electrode contacts simultaneously with a bandpass of $0.1-100 \mathrm{~Hz}$. In most patients, the EEG was sampled continuously at either 4 or $6 \mathrm{msec} /$ point throughout the entire duration of each sentence. Codes identifying the type and onset of each stimulus were incorporated into the data stream for later processing. Averaged event-related field potentials representing the mean response to the ANOMALOUS and to the NORMAL sentenceending words were computed offline.

Particular attention was given to the distribution of voltage over closely spaced electrodes since the voltage amplitude for field potentials falls sharply with increasing distance from the neurons which generate them. Sharp voltage gradients over small distances are, therefore, one means for identifying locally generated fields. Polarity inversions of voltage are particularly useful, as they may indicate that the electrodes span the active neural tissue (Allison et al., 1986).

\section{Results}

Recordings were made from extensive regions of frontal, parietal, and occipital lobe. Focally distributed ERPs that differentiated the ANOMALOUS and NORMAL sentence-ending words were recorded consistently only from temporal lobe sites, particularly the anterior-medial temporal lobe. Figure 1 shows field potentials elicited by ANOMALOUs (solid line) and NORMAL (dashed) sentence-ending words from MT probe electrodes in four patients that are illustrative of the entire patient group. For patients MMN and WSN, the recordings were obtained from the left hemisphere; and in patients KGN and SWD, recordings were obtained from the right hemisphere. The electrode positions for the field potentials shown are indicated by open (MMN, KGN) or closed (WSN, SWD) circles on schematic drawings from Talairach and Szikla (1967). These drawings indicate that the electrode trajectories passed through frontal lobe and terminated in the anterior medial temporal lobe near the amygdala In these patients, electrode locations were determined from skull films taken intraoperatively and projected into the Talairach atlas through the use of bony landmarks. The ERPs are arranged with the most superior electrode at the top of each column, and the most inferior electrode at the bottom.

In each patient, the ANOMALOUs sentence-ending word elicited a large (approximately $50 \mu \mathrm{V}$ ) field potential, which diverged from that elicited by the NORMAL sentence ending at approximately $300 \mathrm{msec}$, and which peaked between 400 and $500 \mathrm{msec}$. This potential had a very focal intracranial distribution restricted to electrodes within the anterior medial temporal lobe. The waveform morphology was similar across patients and was similar for right and left hemispheres. The negative potential was broad with a flat (MMN, LPT1) or bifid minimum (KGN, RPT1).

The negative ERP obtained in patient MMN had a broad, flattened minimum. This was also seen in patient KGN in whom two negative subpeaks were evident. Figure 2 presents data from three additional patients that illustrate the variability in waveshape across patients. Each column represents three AMTL electrodes from a left posterior-temporal probe in each patient. The ERPs at the top of each column were close to the anterior hippocampus, while those at the bottom of the column were located more anteriorly. For patient MMS, two subpeaks were 
Figure 1. ERPs elicited by ANOMALOUS (solid line) and NORMAL sentence-ending words and recorded from electrodes along the left $(L M T)$ or right midtemporal $\left(R M T^{\prime}\right)$ probes are shown superimposed. Each column represents data from a single patient with ERPs from the most superior electrode at the top of each column. This same electrode order is maintained in the inset overlays taken from the atlas of Talairach and Szikla (1967) in which the schematic outlines of the hippocampus and amygdala are shown. Unless otherwise noted, the waveforms in this and all following figures comes from righthanded patients. The data from patients MMN (open circle) and WSN (lefthanded, closed circle) were obtained from the left hemisphere, and the data from patients KGN (open circle) and SWD (closed circle) were obtained from the right hemisphere. The black arrows indicate the AMTL N400s that were distributed focally near the amygdala.
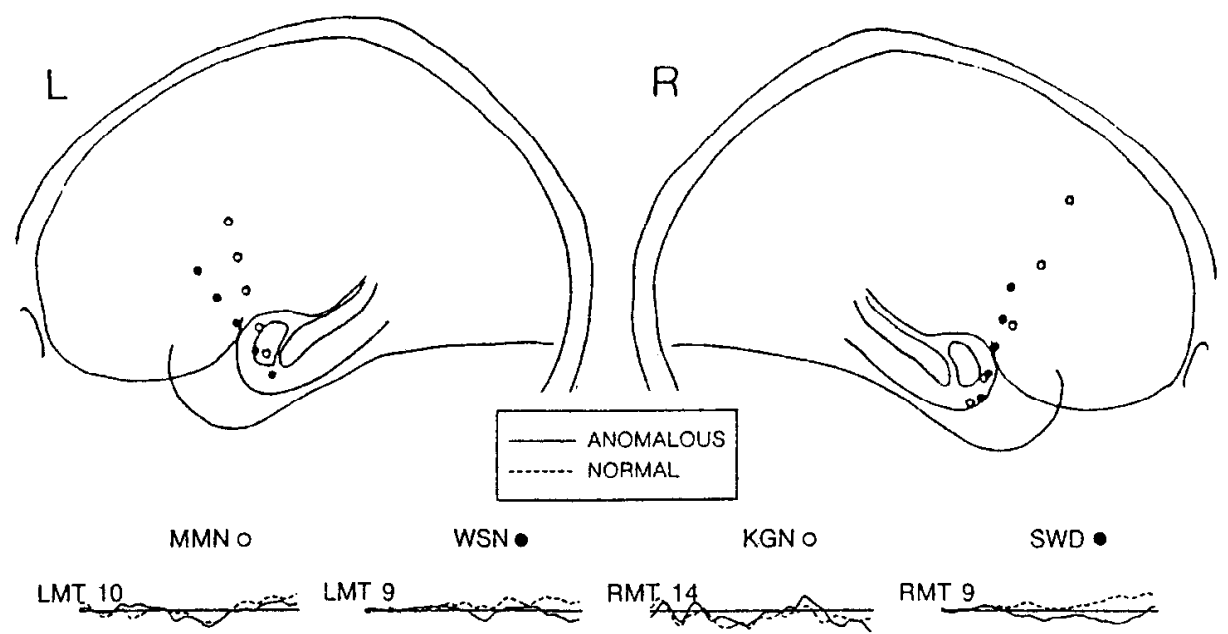

8

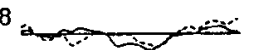

6
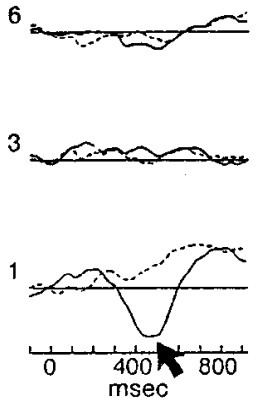

MMS

GAR
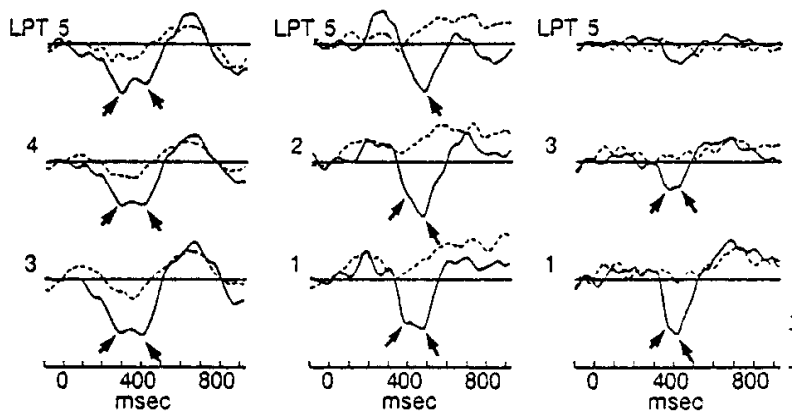

3
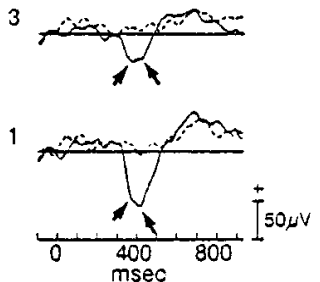

Figure 2. ERPs elicited by ANOMALOus (solid lire) and NORMAL sentemporal probe $(L P T)$ are shown superimposed. All electrodes shown were located anterior to the hippocampus along a straight trajectory. Each column represents data from a single patient arranged such that the electrode nearest the hippocampus is at the top of the column and the electrode at the bottom of each column is at or anterior to the amygdala. The variable and bifid waveshapes of the AMTL N400 are indicated by black arrows.

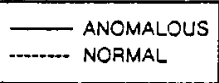
tence-ending words and recorded from electrodes along the left posterior

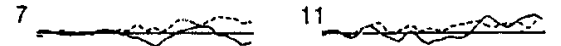

7

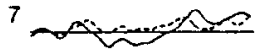

5
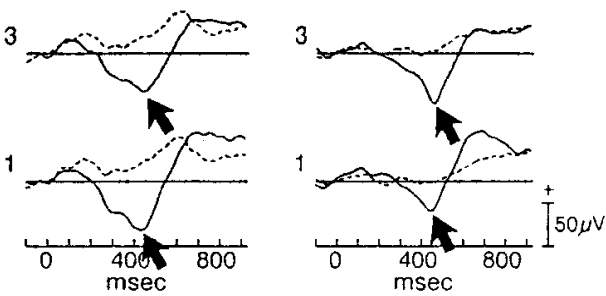

clearly evident, with the earlier peak occurring at about 300 msec and the later peak at $400 \mathrm{msec}$ (see arrows). The subpeaks had somewhat different amplitude distributions, suggesting that this complex potential may reflect either a distributed neural generator within the AMTL, or possibly the activity of two or more discrete generators with overlapping temporal courses. The negative ERPs for patients GAR and RCN also show two distinct subpeaks with differing amplitude distributions within the AMTL. In all three patients, a slow positive potential followed the negative ERP and peaked at 600-700 msec.

The focal, negative AMTL field potential elicited by ANOMALOUS sentence endings was clearly evident in the majority of patients with electrodes within the AMTL (46 of 59 patients with left hemisphere electrodes, and 43 of 58 patients with right hemisphere electrodes). Due to its latency and polarity, we will refer to this negative ERP complex as the AMTL N400. The relationship between the AMTL N400 and the N400 obtained in scalp recordings will be considered in the discussion.

In prior reports (e.g., McCarthy et al., 1989; Paller et al., 1992), we described a negative field potential from electrodes within the hippocampus that was elicited by target stimuli within categorization tasks. These targets could be simple or complex auditory, somatic, or visual stimuli, or even stimulus omissions if the task was suitably structured. Similar target events elicit the P300 ERP in scalp recordings (Sutton et al., 1965; Donchin and Coles, 1988). The relationship between the AMTL N400 and the "hippocampal P300" is considered in Figure 3 in which ERPs for three patients are shown. The ERPs in each column 
A

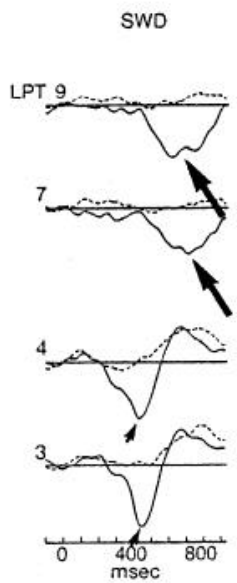

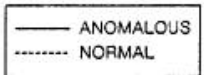
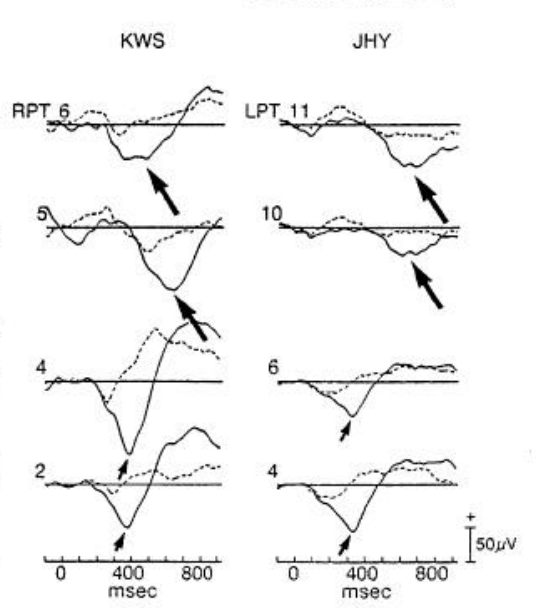

B

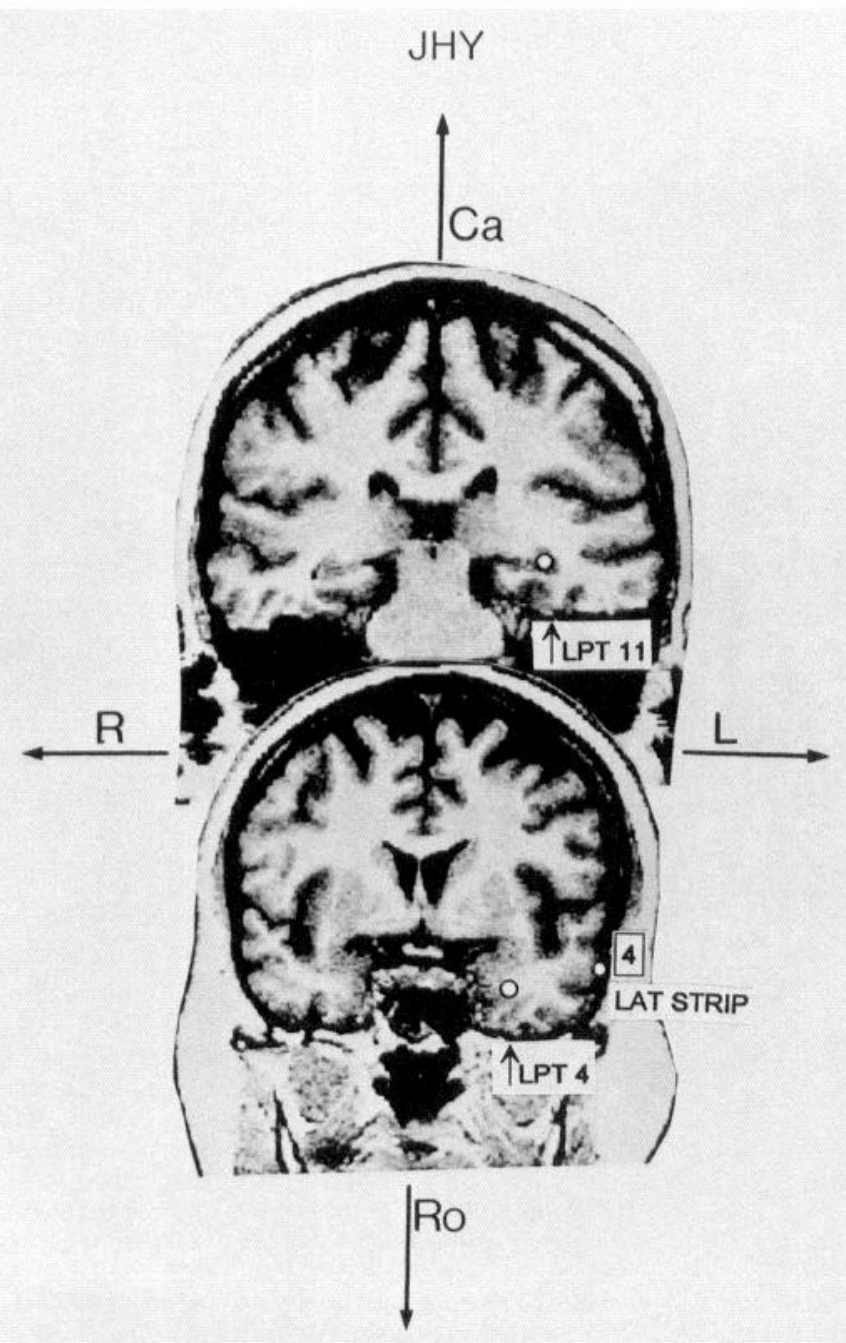

Figure 3. A, ERPs elicited by ANOMALOUS (solid line) and NORMAL sentence-ending words and recorded from electrodes along the left $(L P T)$ or right $(R P T)$ posterior temporal probe are shown superimposed. Each column represents data from a single patient arranged such that the two electrodes at the top of each column were located within the hippocampus, and the two electrodes at the bottom of each column were located anterior to the hippocampus near the amygdala. The small rightpointing arrows at the bottom point to the AMTL N400. The larger, leftpointing arrows at the top point to a longer latency ERP generated in the hippocampus which has been observed in prior studies using categorization tasks (e.g., McCarthy et al., 1989). B, MRI for patient JHY showing electrode locations discussed in Figures $3 A$ and 8. LPTI1 is shown in the upper figure as a white dot with black border located within the hippocampus. LPT4 is shown in the lower figure as a white dot with black border located medially in anterior white matter. LAT4 is shown as a white dot located on the middle temporal gyrus indicated by a small black arrow. In this and all remaining figures, MRIs are oriented in the same way. $\mathrm{Ca}$ : caudal, $R o$ : rostral, $L:$ left, $R$ : right. 
A
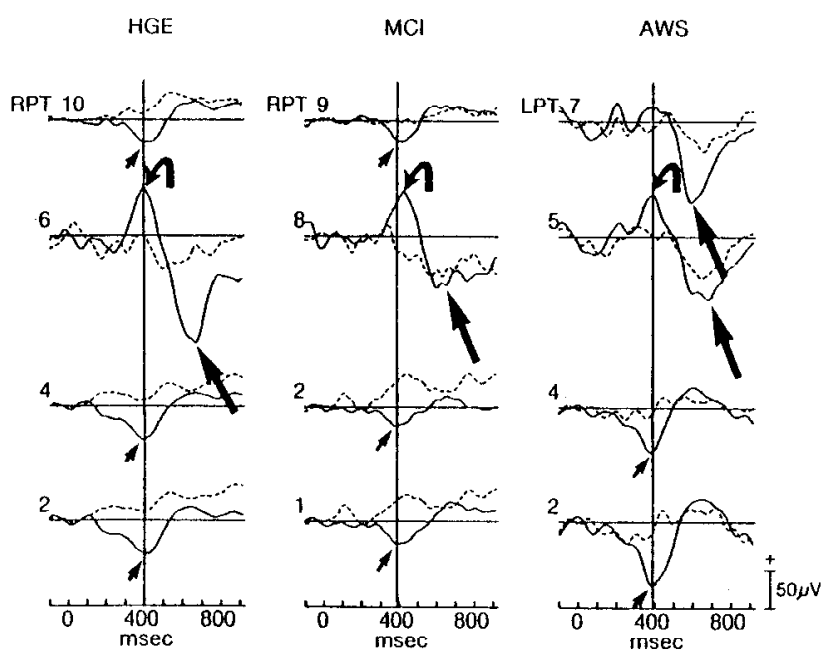

B
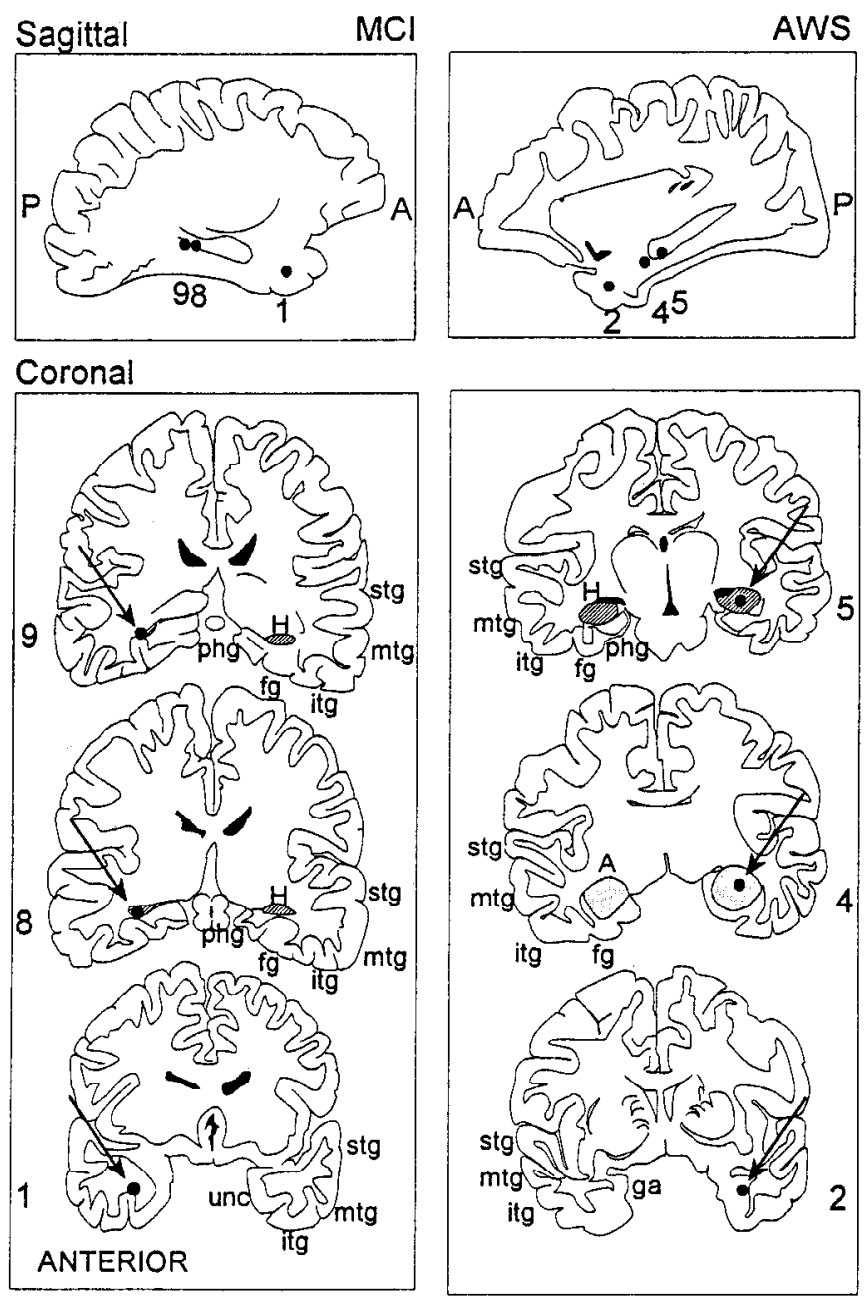

Figure 4. A, ERPs elicited by ANOMALous (solid line) and NORMAL sentence-ending words and recorded from electrodes along the left $(L P T)$ and right $(R P T)$ posterior temporal probes are shown superimposed. Each column represents data from a single patient arranged such that the two electrodes at the top of each column were located within or represent activity in electrodes along either the left (SWD and JHY) or right (KWS) posterior-temporal probes.

In patient SWD, large, focal AMTL N400s were elicited by ANOMALOUs sentence endings at LPT 3 and 4 located anterior to the hippocampus. Longer latency negative ERPs were also elicited by ANOMALOus, but not NORMAL sentence endings at electrodes LPT 7 and 9, which were located within the hippocampus. These later hippocampal field potentials were similar to those obtained in categorization tasks of the type used by McCarthy et al. (1989) in this same patient (not shown). A similar intracranial pattern was obtained for patients KWS and JHY. Like SWD, the AMTL N400 occurred earlier than the hippocampal ERP for both KWS and JHY. The electrode locations for JHY are shown in the MRI inset (Fig. $3 B$ ). These presurgical images were coregistered with postimplant images on which the electrodes were visible. LPT 11 is represented by the small white dot located within the hippocampus of the caudal (Ca) image. LPT4 is represented by the white dot located within the white matter just lateral and inferior to the amygdala in the rostral (Ro) image.

For the patients in Figure 3 and for all other patients, the AMTL N400s occurred earlier in latency than the negative hippocampal ERP that has been associated with P300 (McCarthy et al., 1989). Hippocampal negative ERPs were obtained in 44 posterior-temporal probes (in some instances, the probes did not penetrate the hippocampus). In 25 of these probes, the hippocampal negative ERP was preceded by a sharp positive potential that had a more variable temporal relationship with the AMTL N400. Figure 4 illustrates this point with data from three patients. In patient $\mathrm{HGE}$, a positive-negative hippocampal ERP complex can be seen at RPT 6 between 300 and $800 \mathrm{msec}$. AMTL N400s were observed at RPT 2 and 4, which were located anterior to the hippocampus. In HGE, the latency of the AMTL N400 was the same as the positive peak of the hippocampal positive potential (curved arrow).

The MRI drawings in Figure $4 B$ show the locations of electrodes for MCI and AWS. These images were traced from postimplant MRIs in which electrodes were visible as susceptibility artifacts. For AWS, electrodes LPT 7 and 5 were located within the hippocampus and both show a large, negative hippocampal ERP. At LPT 5, this negative ERP was preceded by a positive ERP, similar to the positive-negative complex observed for HGE at RPT 6. LPT 4 was located a few millimeters anterior to LPT5. Here, an AMTL N400 was observed with the same latency as the hippocampal positive ERP. This polarity inversion suggests that these ERPs might represent activity in the same hippocampal neurons. Note, however, that the AMTL

near the hippocampus, and the two electrodes at the bottom of each column were located anterior to the hippocampus. The small rightpointing black arrows point to the AMTL N400. The left-pointing black arrows point to the longer latency ERP generated in the hippocampus. The curved black arrows point to the positive potential recorded in the hippocampus that formed a biphasic complex with the later hippocampal negative ERP. B. Tracings of MRIs for patients MCI (left column) and AWS (right column) whose ERP data are shown in $A$. A sagittal view ( $A$ : anterior, $P$ : posterior) is presented at the top of each column depicting probe contacts discussed in the text. Coronal views are presented for these same contacts, which are indicated by large arrows. $H$ : hippocampus, $A$ : amygdala, unc: uncus, ga: gyrus ambiens, phg: parahippocampal gyrus, $f g$ : fusiform gyrus, itg: inferior temporal, mtg: middle temporal gyrus, stg: superior temporal gyrus. 

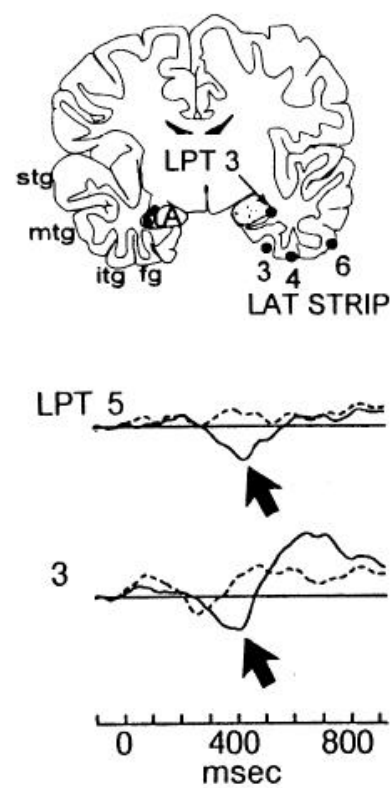

3
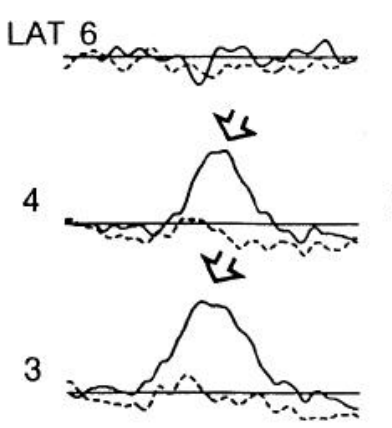

2
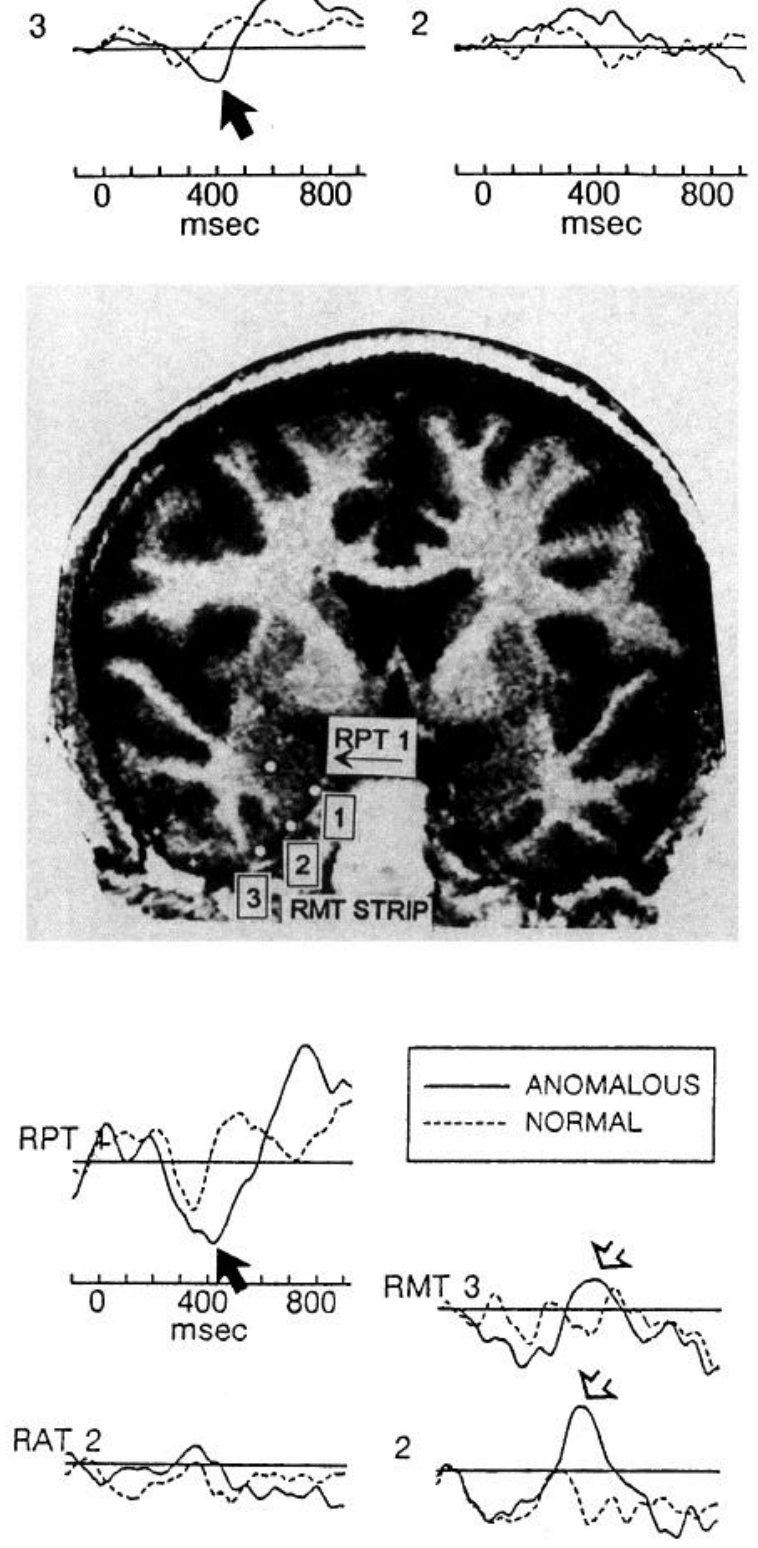

1

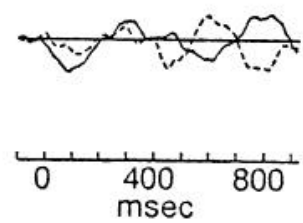

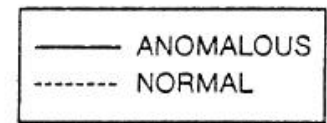

1

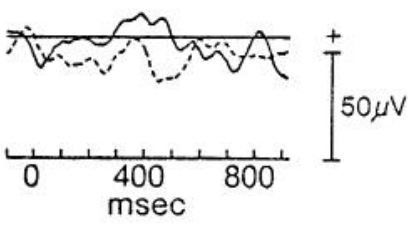

Figure 5. ERPs elicited by ANOMALOus (solid line) and NORMAL sentence-ending words and recorded from electrodes along the left posterior temporal probe $(L P T)$, left anterior temporal $(L A T)$, and left mid-temporal $(L M T)$ subdural strips are shown superimposed. The black arrows point to the AMTL N400, which was recorded from the LPT probe. LPT3, shown on the MRI tracing, was located on the border of the lateral border of the amygdala and is indicated by the labeled arrow. The white arrows point to AMTL P400s which were recorded from LAT and LMT subdural strips. The locations of LAT3-4 and 6 are depicted on the brain surface. LMT 1-3 were positioned slightly posterior to LAT contacts and are not shown in the tracing.

$\mathrm{N} 400$ is larger in amplitude at an even more anterior site at LPT 2, located in white matter anterior to the amygdala.

For MCI, RPT 8 was located within the body of the hippocampus and showed a positive-negative complex similar to HGE's hippocampal recording at RPT 6. An AMTL N400 was recorded from RPT 1, which was located just superior to the collateral sulcus. Unlike the data for patients HGE and AWS, the AMTL N400 had a peak latency that was approximately 50 msec earlier than the positive ERP recorded from within the hippocampus.

In HGE (RPT 10) and MCI (RPT 9), a negative ERP with the same latency as the AMTL N400, but with somewhat smaller amplitude, was observed. For MCI, this electrode was located posterior to the hippocampus, and just superior to the collateral sulcus.

Subdural electrode strips were often implanted in addition to depth probes. In some patients, simultaneous recordings were made from depth electrodes and electrodes on temporal neocortex. Figure 5 presents ERP recordings from a patient in whom an AMTL N400 was recorded from left posterior-temporal probe (LPT) electrode 3 indicated by a black arrow at the lateral border of the amygdala in the MRI tracing. An electrode strip encircled the temporal neocortex with electrodes LAT 3 indicated on the parahippocampal gyrus just medial to the collateral sulcus, and LAT 4 on the crown of the fusiform gyrus. LAT 6 was located on the inferior temporal gyrus. Large, focal positive ERPs with peak latencies of approximately $400 \mathrm{msec}$ were elicited by the

\footnotetext{
Figure 6. ERPs elicited by ANOMALOUs (solid line) and NORMAL sentence-ending words and recorded from electrodes along the right posterior temporal probe $(R P T)$ and right anterior temporal $(R A T)$ and right midtemporal $(R M T)$ subdural strips are shown superimposed. The black arrow points to the AMTL N400, which was recorded from the anterior RPT probe. RPTI, shown on the MRI, was located in the basolateral amygdala and is indicated by the labeled arrow. The white arrows point to AMTL P400s, which were recorded from the RMT2 and 3 (indicated by dots and numbered boxes) which straddled the collateral sulcus. P400 was not recorded from RMT1, located more medially, nor from RAT1-2, located more anteriorly (not depicted on MRI).
} 

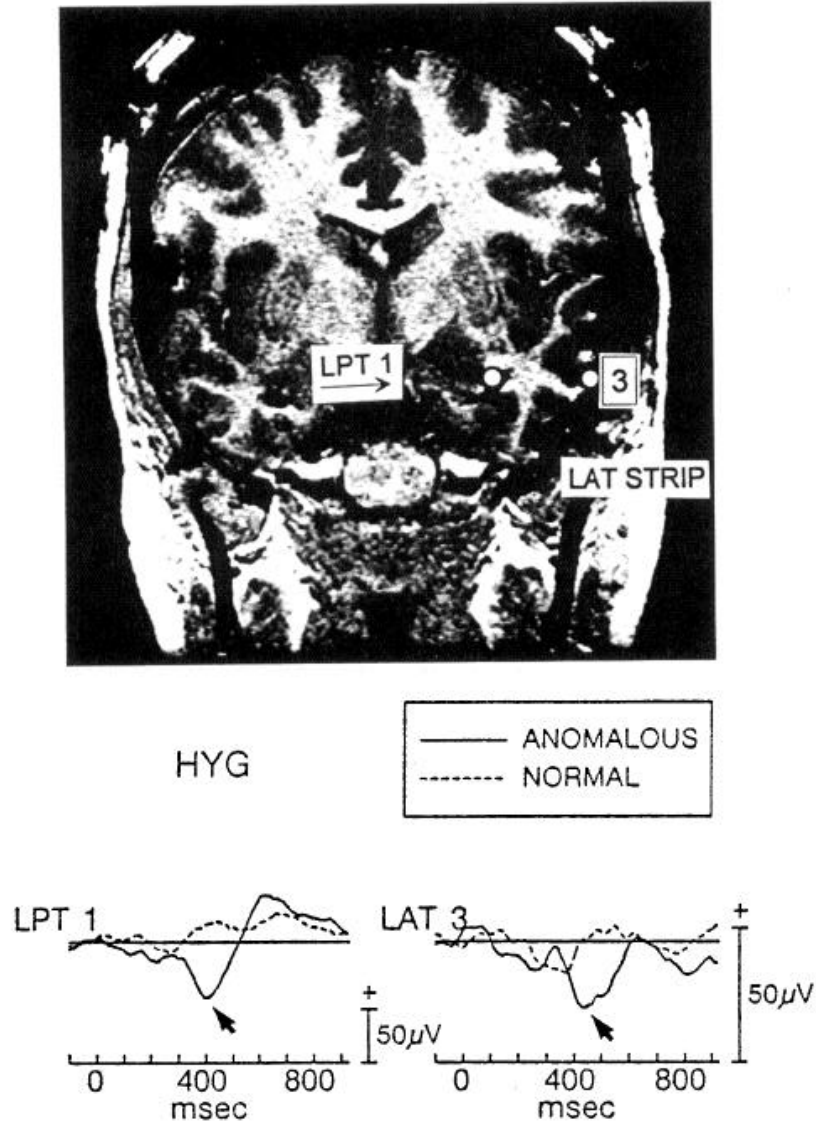

Figure 7. ERPs elicited by ANOMALOus (solid line) and NORMAL sentence-ending words and recorded from the most anterior electrode of the left posterior temporal probe $(L P T I)$ and from left anterior subdural strip contact 3 (LAT3) are shown superimposed. LPT1 was located in white matter lateral to the amygdala (indicated by dot and labeled arrow) and LAT3 was located on the middle temporal gyrus (dot and numbered box). Black arrows indicate the AMTL N400s. Note difference in amplitude calibration.

ANOMALOUS sentence endings at LAT 3 and 4, but not more laterally at LAT 6 , or more medially at LAT 2 . Similar results were obtained for a more posterior subdural strip where a positive $400 \mathrm{msec}$ ERP was obtained at LMT2 (just posterior to LAT 4, location not shown).

A similar pattern was obtained in the right hemisphere of another patient depicted in Figure 6. An AMTL N400 was obtained at RPT 1 within the amygdala. A positive potential

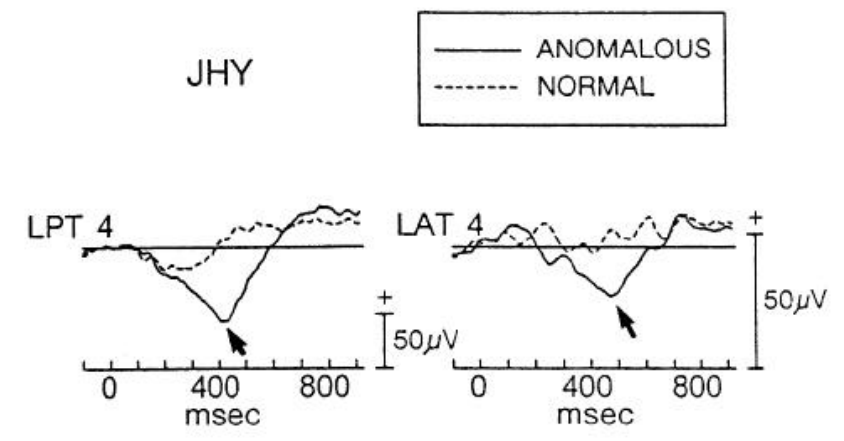

Figure 8. ERPs elicited by anOMalous (solid line) and NORMaL sentence-ending words and recorded from electrode 4 of the left posterior temporal probe (LPT4) and from left anterior subdural strip contact 4 (LAT 4) are shown superimposed. Black arrows indicate the AMTL N400s. $L P T 4$ was located lateral to the amygdala and $L A T 4$ was located on the middle temporal gyrus. Note difference in amplitude calibration. Electrode locations are depicted in the MRI shown in Figure 3.

with a $400 \mathrm{msec}$ latency (AMTL P400) was recorded at RMT 2 and 3 , which straddled the collateral sulcus. The amplitude of the positive ERP was smaller at a more medial location (RMT 1). The subdural strip just anterior to RMT showed very little if any AMTL P400 activity (RAT 2), indicating the focal distribution of the potential.

While positive ERPs were obtained over fusiform and parahippocampal gyri, smaller negative ERPs were obtained from more lateral neocortical sites. Data for patient HYG are shown in Figure 7 where a large AMTL N400 was recorded from the white matter lateral to the amygdala. At LAT 3 on the middle temporal gyrus, a smaller negative ERP was simultaneously recorded. Figure 8 presents data from patient JHY, whose MRI is shown in Figure 3. A large AMTL N400 was recorded from LPT 4 lateral to the amygdala. A smaller negative ERP with the same latency was recorded at LAT 4, located on the middle temporal gyrus.

The data for patient KSR presented in Figure 9 further illustrates several of the preceding points. Large AMTL N400s were obtained from LPT 1, 2, and 3. LPT 3 was located in the lateral part of the amygdala, while LPT 2 was located above the collateral sulcus. Subdural strip electrodes LAT 2 and 3 were located near the collateral sulcus, and both recorded small AMTL P400s. LAT 6, on the inferior temporal gyrus, recorded a small negative ERP at 400 ms. LPT 6, within the body of the hippocampus, recorded a positive-negative complex similar to pa-

Table 1. Average locations of intracranial ERPs using coordinates of Talairach and Tournoux (1988)

\begin{tabular}{|c|c|c|c|c|c|c|c|}
\hline & \multirow[b]{2}{*}{$N$} & \multicolumn{2}{|l|}{$\mathrm{X}$} & \multicolumn{2}{|l|}{$\mathrm{Y}$} & \multicolumn{2}{|l|}{$\mathrm{Z}$} \\
\hline & & Median & Range & Median & Range & Median & Range \\
\hline Left AMTL N400 & (17) & E. 00 & C. 70 -E. 30 & lb. 66 & lb.11-lc. 01 & 10.99 & $10.39-11.95$ \\
\hline Right AMTL N400 & (11) & E. 15 & D.43-E.73 & rb. 53 & rb. $42-r b .81$ & 11.03 & $9.98-11.86$ \\
\hline Left Hipp. P300 & (7) & F.15 & E.60-F.42 & lb. 65 & lb.55-lb.76 & 9.13 & $8.61-10.05$ \\
\hline Right Hipp. P300 & (7) & F.04 & E. 73-F.22 & rb. 55 & rb.44-rb. 74 & 9.53 & $9.03-10.27$ \\
\hline Left AMTL P400 & $(8)$ & E. 37 & C.78-F.64 & lb. 54 & lb.15-lc. 76 & 11.66 & $9.97-12.63$ \\
\hline Right AMTL P400 & (8) & E. 30 & E. $28-F .79$ & rb. 89 & rb.33-rc. 69 & 12.18 & $10.30-12.86$ \\
\hline
\end{tabular}

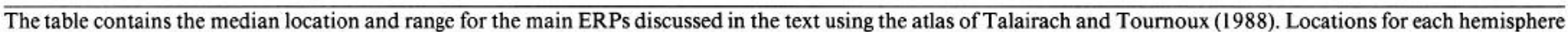

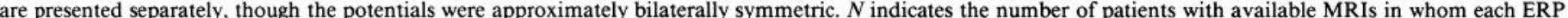

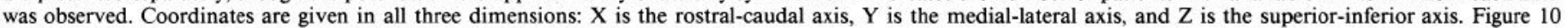
presents a graphical depiction of the median Talairach location for each ERP. 

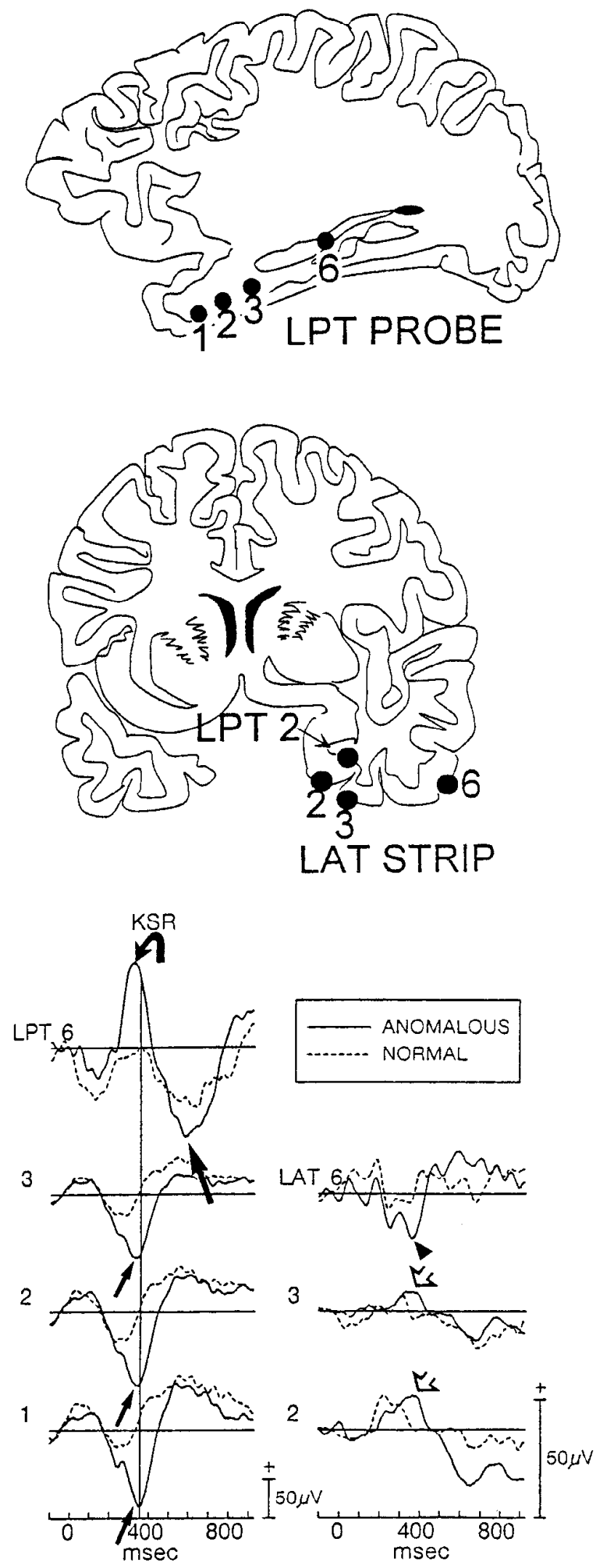

Figure 9. ERPs elicited by ANOMALOus (solid line) and NORMAL sentencc-ending words and rccorded from the left postcrior tcmporal probe $(L P T)$ and from the left anterior temporal subdural strip are shown superimposed. Small, right pointing black arrows indicate the AMTL N400s at LPT1-3. The curved and left pointing arrows indicate the hippocampal biphasic complex at LPT6. The open arrows indicate small tients HGE, MCI, and AWS (Fig. 6). Note that in this instance, the positive hippocampal ERP peaked approximately $50 \mathrm{msec}$ prior to the AMTL N400.

Table 1 summarizes the locations from which the AMTL N400, AMTL P400, and hippocampal P300 (negative ERP) were recorded using the coordinates of the allas of Talairach and Tournoux (1988). The median locations are depicted graphically in Figure 10. The median location for AMTL N400 occurred at the anterior border of the amygdala, while the median location for the hippocampal P300 occurred in the midbody of the hippocampus. The median location for the AMTL P400 occurred just lateral to the collateral sulcus in the anterior fusiform gyrus and was just inferior to the median location of the AMTL N400. The distribution for each ERP was approximately bilaterally symmetric.

\section{Discussion}

A negative-polarity field potential with a peak latency between 400 and $500 \mathrm{msec}$ and with a focal intracranial distribution within the anterior-medial temporal lobe was consistently elicited by ANOMALOUS, but not by NORMAL, sentence-ending words. These AMTL N400s were largest in amplitude anterior to the hippocampus, and were obtained from sites within the amygdala and the more lateral white matter superior to the collateral sulcus. No polarity inversions or sharp amplitude gradients were observed between closely spaced electrodes within and nearby the amygdala, making it unlikely that neurons within the amygdala generated the AMTL N400.

Polarity inversions were noted, however, between subdural electrodes over the anterior fusiform and parahippocampal gyri near the collateral sulcus and the white matter just superior to these sites. Negative field potentials were recorded from depth electrodes within the AMTL while large, focal, positive potentials were recorded from the underlying neocortical surface in the region of collateral sulcus (see Figs. 5, 6, 10). This spatial distribution suggests that all or part of the AMTL N400 is generated by neocortex near the collateral sulcus, including the anterior fusiform and, perhaps, parahippocampal gyri. If this hypothesis is correct, negative potentials should be recorded from locations superior to this region that would diminish in amplitude with increasing distance from the collateral sulcus. That small amplitude negative potentials were recorded at more lateral and superior neocortical sites on the inferior and middle temporal gyri (see Figs. 7-9) was consistent with this interpretation.

In some patients, we noted that negative ERPs similar to the AMTL N400 were recorded from electrodes in white matter superior to the posterior fusiform gyrus and collateral sulcus and posterior to the hippocampus (see Fig. 4, RPT 10 for HGE and RPT 9 for $\mathrm{MCI}$ ). Whether this represents an additional focus of activation, evidence for an extended neural source along the collateral sulcus, or simply volume conduction from the anterior temporal lobe, cannot be distinguished by the present data. We have not observed focal positive ERPs with similar

amplitude AMTL P400s at LAT2-3, and a black triangle indicates a small amplitude AMTL N400 at LAT6. Electrode contacts are indicated on sagittal and coronal MRI tracings. The sagittal view (top) shows the locations of the probe contacts relative to the hippocampus. The coronal view (bottom) shows the location of the subdural contacts relative to the depth electrode LPT2. 


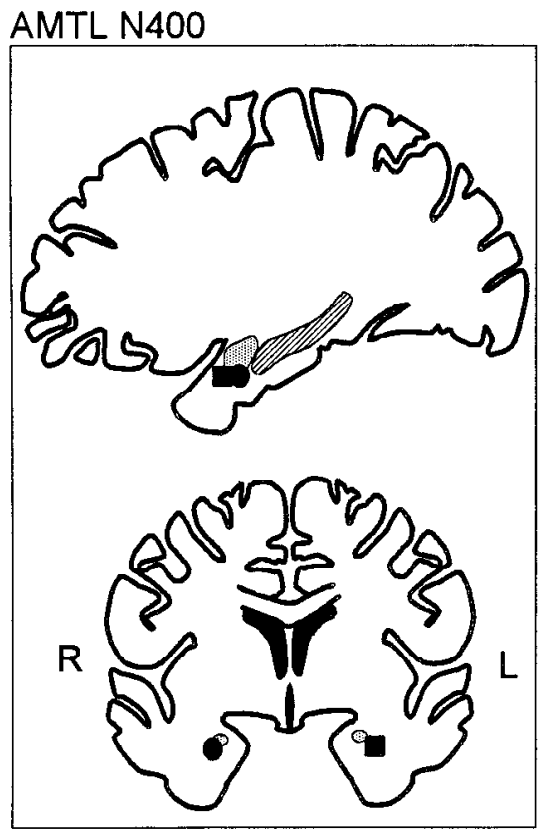

Hippocampal P300

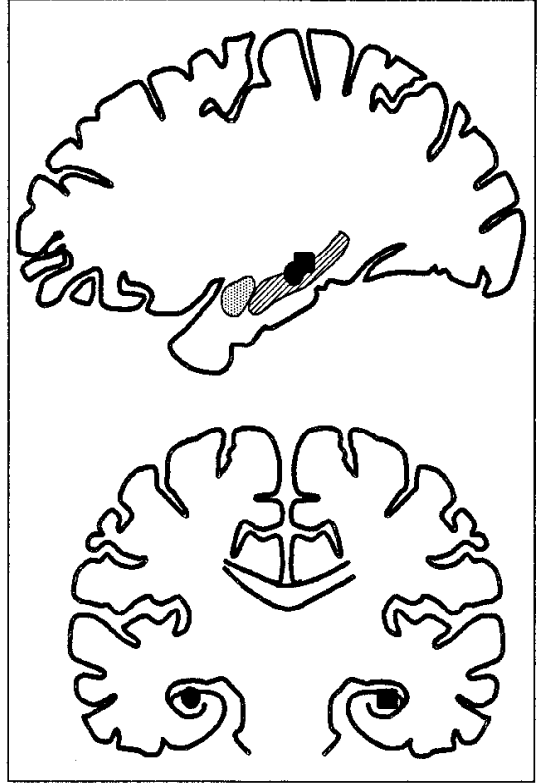

AMTL Surface P400

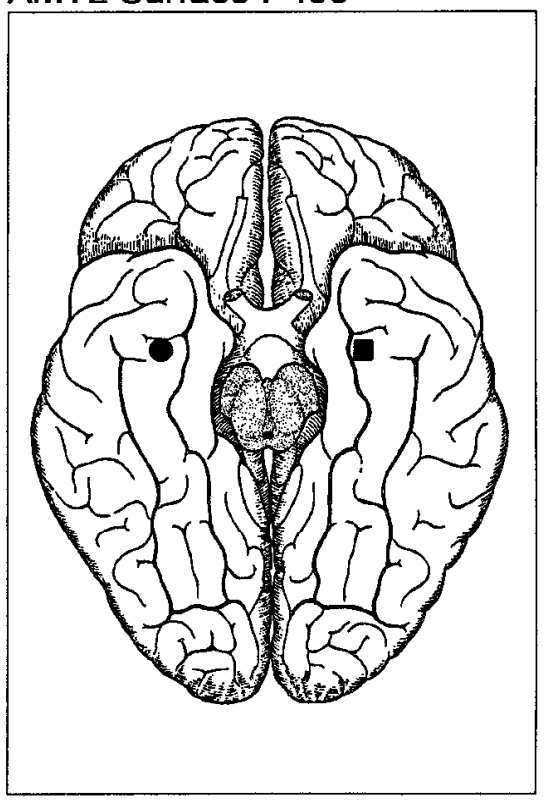

Figure 10. Median Talairach locations for the ERPs discussed in the text are depicted. Left and right hemisphere locations are indicated by squares and circles, respectively. Locations of ERPs recorded from depth probes are shown using sagittal and coronal planes. Locations of cortical surface ERPs are shown on the inferior surface of the brain. AMTL N400, shown in the left column, was located in the anterior border of the amygdala. Hippocampal P300, shown in the middle column, was located within the midbody of the hippocampus. The cortical surface AMTL P400, shown in the right column, was located laterally to the colateral sulcus in the anterior fusiform gyrus.

latency from subdural electrodes in this region, and the negative potentials in white matter have been smaller and less focally distributed than the AMTL N400s.

AMTL N400s were obtained in both left and right hemisphere recordings (e.g., see Fig. 1). It is difficult to draw strong conclusions about the interhemispheric amplitude distribution of the AMTL N400 because small differences in electrode position can result in large amplitude variations and patients often had lateralized temporal lobe pathology. Nevertheless, no obvious trend for an interhemispheric amplitude asymmetry was noted.

\section{Relationship to hippocampal field potentials}

Our conclusion that the AMTL N400s were generated in the neocortex near the collateral sulcus is complicated by the presence of temporally overlapping potentials of apparent hippocampal origin. In addition to the AMTL N400, ANOMALOus sentence endings often, but not always, elicited a large negative field potential in the hippocampus (see Figs. 3, 4). This hippocampal field potential had a similar amplitude and intracranial distribution as the hippocampal ERPs elicited by targets in categorization tasks using simple nonverbal stimuli (McCarthy et al., 1989). In all patients, the AMTL N400 occurred at an carlier latency than this large hippocampal negative field potential.

In most patients (e.g., see Fig. 3), the large negative hippocampal ERP was monophasic; however, in some patients, the hippocampal negative ERP was preceded by a positive ERP which formed a biphasic complex (see Fig. 4). The earlier positive hippocampal ERP had a variable latency relationship with the AMTL N400. For example, in patient AWS, the AMTL N400 at LPT 4 was very similar in waveshape and latency, but opposite in polarity, to the hippocampal complex recorded a few millimeters posteriorly. This suggests that some of the AMTL
N400 may represent one side of a hippocampal "dipole." This conclusion is inconsistent, however, with the data from patient MCI where these two ERPs had different peak latencies. Also, in most patients, including AWS, the largest AMTL N400s were not obtained at the electrodes closest to the anterior hippocampus, but more at sites more anterior. However, as emphasized in Figures 1 and 2, the AMTL N400 itself often has a complex waveshape with two negative subpeaks. Part of this complex waveshape may represent volume-conducted activity from the hippocampus.

The amplitude of the task-related hippocampal ERPs is strongly affected by hippocampal pathology and has been used clinically to help detect the focal, epileptogenic hippocampus in intracranial recordings (McCarthy et al., 1987). In contrast, we have not found that the AMTL N400 is useful in predicting hippocampal pathology, suggesting that the AMTL N400 is not generated entirely by the same hippocampal neurons that generate the hippocampal ERPs.

In summary, the data suggest that the AMTL N400 primarily represents activity in inferior temporal neocortex near the collateral sulcus, including the anterior fusiform and parahippocampal gyri. A more precise anatomical specification is not warranted by the present data. The AMTL N400 is physiologically distinct from later hippocampal negative field potentials elicited by ANOMALOUs sentence endings. The preceding positive hippocampal ERP that temporally overlaps the AMTL N400 may reflect activity in a hippocampal population, which also has a negative field component outside the hippocampus and which may contribute to the amplitude of the AMTL N400.

Overall, the data indicates that the AMTL N400 and the hippocampal field potentials reflect activity in distinct neuronal populations. The generators, however, are in close spatial proximity. Furthermore, the hippocampus and the neocortex in in- 
ferior AMTL may be functionally related in these language tasks. Both the proximity and the anatomic interconnections contribute to the complex spatial and temporal overlap of the two field potentials.

The scalp distribution of N400 in sentence tasks is complex and likely represents temporally overlapping activity from different neural generators. One component of the distribution, however, has a broad midline central distribution at $400 \mathrm{msec}$, suggestive of a deep neural generator (Kutas et al., 1988; Nobre and McCarthy, 1994). The AMTL N400 has a similar latency as the scalp-recorded N400 and is similarly affected by the same anomalous sentence task. The 3676 electrode sites sampled in this study included virtually the entire cortical mantle. No region outside of the AMTL consistently produced large field potentials of the appropriate latency in this task, strongly implicating this region as one of the neural generators of the scalp-recorded N400.

The N400 obtained in scalp recordings is sensitive to tasks in which word type and word priming by semantic associates is varied. In the following article (Nobre and McCarthy, 1995), these task manipulations are investigated to test further the relationship between the AMTL N400 and the N400 obtaincd in scalp recordings.

\section{References}

Allison T, Wood CC, McCarthy G (1986) The central nervous system. In: Psychophysiology (Coles MGH, Donchin E, Porges SW, eds), pp 5-25. New York: Guilford.

Allison T, Ginter H, McCarthy G, Nobre AC, Puce A, Luby M, McCarthy K, Spencer DD (1994) Face recognition in the human extrastriate cortex. J Neurophysiol 71:821-825.

Barrett SE, Rugg MD (1989) Event-related potentials and the semantic matching of faces. Neuropsychologia 27:913-922.

Barrett SE, Rugg MD (1990) Event-related potentials and the semantic matching of pictures. Brain Cogn 14:201-212.

Bentin S, McCarthy G, Wood CC (1985) Event-related potentials, lexical decision, and semantic priming. Electroencephalogr Clin Neurophysiol 60:343-355.

Darcey TM, Williamson PD (1985) Spatio-temporal EEG measures and their application to human intracranially recorded seizures. Electroencephalogr Clin Neurophysiol 61:573-587.

Donchin E, Coles MGH (1988) Is the P300 component a manifestation of context updating? Behav Brain Sci 11:357-374.

Fischler I, Raney GE (1989) Language by eye: behavioral, autonomic, and cortical approaches to reading. In: Handbook of cognitive psychology: central and autonomic nervous system (Jennings JR, Coles MGH, eds). New York: Wiley.

Halgren E, Squires NK, Wilson CL, Rohrbaugh JW, Babb TL, Crandall PH (1980) Endogenous potentials generated in the human hippocampal formation and amygdala by infrequent events. Science 210: 803-805.

Holcomb PJ (1993) Semantic priming and stimulus degradation: im- plications for the role of the N400 in language processing. Psychophysiology 30:47-61.

Holcomb PJ, McPherson WB (1994) Event-related potentials reflect semantic priming in an object decision task. Brain Cogn 24:259-276.

Kutas M, Hillyard SA (1980) Event-related brain potentials to semantically inappropriate and surprisingly large words. Biol Psychol 11:99-116.

Kutas M, Hillyard SA (1989) An electrophysiological probe of incidental semantic association. J Cogn Neurosci 1:38-49.

Kutas M, Van Petten C (1988) Event-related potential studies of language. In: Advances in psychophysiology (Ackles PJ, Jennings JR, Coles MGH, eds), pp 139-197. Greenwich: JAI.

Kutas M, Hillyard SA, Gazzaniga MS (1988) Processing of semantic anomaly by right and left hemispheres of commissurotomy patients. Brain 111:553-576.

McCarthy G, Wood CC (1984) Intracranially recorded event-related potentials during sentence processing. Soc Neurosci Abstr 10:847.

McCarthy G, Wood CC, Bentin S (1985) Human intracranial ERPs during lexical decision. Soc Neurosci Abstr 11:880.

McCarthy G, Darcey TM, Wood CC, Williamson PD, Spencer DD (1987) Asymmetries in scalp and intracranial endogenous ERPs in patients with complex partial epilepsy. In: Fundamental mechanisms of human brain function (Engel J Jr, Ojemann GA, Luders HO, Williamson PD, eds), pp 51-59. New York: Raven.

McCarthy G, Wood CC, Williamson PD, Spencer DD (1989) Taskdependent field potentials in human hippocampal formation. J Neurosci 9:4253-4268.

McCarthy G, Spencer DD, Riker RJ (1991) The stereotaxic placement of depth electrodes in epilepsy. In: Epilepsy surgery (Luders H, ed), pp 385-393. New York: Raven.

Neville H, Kutas M, Chesney G, Schmidt AL (1986) Event-related brain potentials during initial encoding and recognition of congruous and incongruous words. J Mem Lang 25:75-92.

Nobre AC, McCarthy G (1992) Electrophysiological measures of semantic processing in humans. Soc Neurosci Abstr 18:584.

Nobre AC, McCarthy G (1994) Language-related ERPs: modulation by word type and semantic priming. J Cogn Neurosci 6:233-255.

Nobre AC, McCarthy G (1995) Language-related field potentials in the anterior-medial temporal lobe: II. effects of word type and semantic priming. J Neurosci 15:1090-1098.

Paller KA, McCarthy G, Roessler E, Allison T, Wood CC (1992) Potentials evoked in human and monkey medial temporal lobe during auditory and visual oddball paradigms. Electroencephalogr Clin Neurophysiol 84:269-279.

Rugg MD, Doyle MC (1992) Event-related potentials and stimulus repetition in direct and indirect tests of memory. In: Cognitive electrophysiology (Heinze H, Munte T, Mangun R, eds). Boston: Birkhauser.

Smith ME, Stapleton JM, Halgren E (1986) Human medial temporal lobe potentials evoked in memory and language tasks. Electroencephalogr Clin Neurophysiol 63:145-149.

Sutton SM, Braren M, Zubin J, John ER (1965) Evoked potential correlates of stimulus uncertainty. Science 150:1187-1188.

Talairach J, Szikla G (1967) Atlas of stereotaxic anatomy of the telencephaplon. Paris: Masson.

Talairach J, Tournoux P (1988) Co-planar stereotaxic atlas of the human brain. New York: Thieme. 